\title{
Regulation of Hypoxia-Induced Cell Death in Human Tenocytes
}

\author{
Min Liang, 1, 2 Hannah R. Cornell, ${ }^{1}$ Nasim Zargar Baboldashti, ${ }^{1,3}$ \\ Mark S. Thompson, ${ }^{1,3}$ Andrew J. Carr, ${ }^{1,4}$ and Philippa A. Hulley ${ }^{1}$ \\ ${ }^{1}$ Botnar Research Centre, Nuffield Department of Orthopaedics, Rheumatology and Musculoskeletal Sciences, University of Oxford, \\ Oxford OX3 7LD, UK \\ ${ }^{2}$ Department of Periodontology, Guanghua School of Stomatology and Hospital of Stomatology, Sun Yat-sen University, \\ 56 Lingyuan Road West, Guangzhou 510055, China \\ ${ }^{3}$ Institute of Biomedical Engineering, University of Oxford, Old Road Research Campus, Oxford OX3 7DQ, UK \\ ${ }^{4}$ The Nuffield Department of Orthopaedics, Rheumatology and Musculoskeletal Sciences (NDORMS), NIHR Musculoskeletal BRU, \\ University of Oxford, Oxford OX3 7LD, UK
}

Correspondence should be addressed to Min Liang, liangm8@mail.sysu.edu.cn

Received 3 August 2012; Revised 4 November 2012; Accepted 5 November 2012

Academic Editor: Robert Gillespie

Copyright () 2012 Min Liang et al. This is an open access article distributed under the Creative Commons Attribution License, which permits unrestricted use, distribution, and reproduction in any medium, provided the original work is properly cited.

Degenerate shoulder tendons display evidence of hypoxia. However tendons are relatively avascular and not considered to have high oxygen requirements and the vulnerability of tendon cells to hypoxia is unclear. Cultured human tenocytes were exposed to hypoxia and the cellular response detected using QPCR, Western blotting, viability, and ELISA assays. We find that tenocytes respond to hypoxia in vitro by activating classical HIF- $1 \alpha$-driven pathways. Total hypoxia caused significant tenocyte apoptosis. Transcription factors typically involved in hypoxic response, HIF- $1 \alpha$ and FOXO3A, were upregulated. Hypoxia caused sustained upregulation of several proapoptotic proteins known to mediate hypoxia-induced apoptosis, such as Bnip3 and Nix, but others were unchanged although they were reportedly hypoxia-sensitive in other cell types. Antiapoptotic proteins $\mathrm{Bcl} 2$ and $\mathrm{Bcl}-\mathrm{xL}$ were unchanged by hypoxia. Normal human tenocytes expressed all isoforms of the hypoxia-induced vascular growth factor VEGF except VEGF-D. Hypoxia markedly upregulated VEGF-A mRNA, followed by increased VEGF protein secretion. However treatment with VEGF did not improve tenocyte survival. As a protective strategy for tenocytes at risk of hypoxic death we added prosurvival growth factors insulin or platelet rich plasma (PRP). Both agents strongly protected tenocytes from hypoxia-induced death over $48 \mathrm{~h}$, suggesting possible efficacy in the acute postrupture tendon or integrating graft.

\section{Introduction}

The tissue-specific physiology of tendon is adjusted to extreme mechanical loading, which results in acute and repetitive reductions in blood perfusion and therefore a likely ability to tolerate transient hypoxia. With increasing age tendons are also at increasing risk of degeneration and tear or rupture with the inevitable disruption of blood supply. Tendons such as the rotator cuff in the shoulder are prone to a progressive degenerative process leading to tears in as many as $54 \%$ of the population aged above 60 [1]. These injuries are disabling, painful, and heal poorly. In most cases, the causes of tendon weakness and degeneration are not known and in particular the role of hypoxic insults is not understood.
The enthesis is poorly vascularised in all tendons as is the so-called critical zone, just proximal to the tendon/bone attachment, where the majority of rotator cuff tears take place [2-4]. However, tendon itself is not without blood vessels and there is evidence that considerable vascular remodeling takes place during tendon healing. The vessel count in early stage rotator cuff tears increases by up to 4fold in stark contrast with end-stage degenerative cuff tears which have lower vessel counts than uninjured tendon $[5,6]$. Despite its relatively avascular appearance, tendon is more oxygen-dependent than other joint tissues such as cartilage and requires enhanced perfusion during repair [7].

Metabolically tendon is described as reliant on oxidative phosphorylation during development and early growth with a shift towards anaerobic glycolysis as the tissue matures [7]. 
Following injury tenocytes become more metabolically active with a marked increase in oxidative phosphorylation during the repair process [7]. Given this ability to adapt to changing metabolic demands and the low energy requirements of mature tenocytes it is sometimes assumed that tendon is resistant to changes in blood supply and oxygen level.

Recent studies have however found evidence of classical hypoxia response pathways in tendon $[8,9]$. Torn rotator cuffs express high levels of HIF-1alpha (HIF-1 $\alpha$ ) as well as at least two HIF-driven genes, vascular endothelial growth factor (VEGF) [6] and the proapoptotic Bcl2 family member Bnip3 $[8,9]$. The forkhead transcription factor FOXO3A has also been reported to be upregulated in response to hypoxia [10] and to strongly regulate Bnip3 [11]. Expression of Bnip3 precedes and correlates with increasing levels of apoptotic cell death in advancing stages of rotator cuff degeneration [8]. These data suggest that hypoxia may be a relevant damage factor in tendon injury and that appropriate vascular response may be essential for normal repair and remodeling. However the expression of VEGF is also increased by inflammation or mechanical load [12] and Bnip3 is not exclusively regulated by hypoxia $[11,13]$. It is not clear from these studies what role hypoxia plays in driving this response or how involved the tenocytes themselves are rather than surrounding tissue such as bursa or blood vessels.

Exogenous growth factors are potentially useful agents for the enhancement of tendon healing. VEGF and IGF have been reported to protect cells such as neurons from hypoxic insult $[14,15]$ and IGF has also been reported to protect tenocytes against hypoxia [16]. Similarly second messengers downstream of growth factor receptors such as cAMP can mimic this prosurvival effect [17-19]. Platelet rich plasma (PRP) is currently undergoing investigation as an autologous growth factor therapy in many tissues including tendon [20]. Platelet rich plasma is, by definition, a volume fraction of the plasma, having a platelet concentration above baseline (whole blood) [21]. Platelets are active participants in different healing processes in the body [22]. Platelet $\alpha$-granules are rich in growth factors and other active molecules that have significant roles in facilitating healing. Growth factors released by platelets upon platelet activation include plateletderived growth factor (PDGF), endothelial growth factor (EGF), insulin-like growth factor (IGF-I), transforming growth factor- $\beta$ I (TGF- $\beta$ I), and vascular endothelial growth factor (VEGF) $[22,23]$. These growth factors and many other proteins secreted by platelets are active in the healing cascade by promoting cell proliferation and migration, synthesis of extracellular matrix proteins such as collagen and supporting angiogenesis and vascularisation.

PRP has shown activity in tenocytes and healing tendons although no studies have been performed to establish efficacy against hypoxic damage. Schnabel et al., have found an increase in collagen type I gene expression in cultured tendon cells treated with PRP [24]. In an animal study, greater maturation in tendon callus has been reported after using PRP for augmentation of rat Achilles tendon tears. The same study has also reported higher ultimate stress and increased force to failure in PRP-treated animal tendons [25]. In a recent study, the mobilization of circulation-derived cells was enhanced in the area of PRP injection. They also reported an increase in the production of type I collagen and macrophage proliferation at 3 and 7 days [26]. However direct prosurvival effects of PRP have not yet been demonstrated on tenocytes and we have therefore compared PRP to insulin, VEGF and cAMP in treatment of hypoxic human tenocytes.

We hypothesize that tenocytes are vulnerable to hypoxiainduced cell death and that growth factors may offer a degree of protection, in line with effects on other cell types. We have therefore set out to characterize the response of normal human tenocytes to acute hypoxia ( $0.1 \%$ oxygen) in both high and low serum cultures, to assess the capacity of tenocytes themselves to release proangiogenic factors and to devise protective strategies to enhance tenocyte survival after ischemic insult.

\section{Materials and Methods}

All chemicals and reagents were obtained from Sigma (Poole, UK) except where otherwise stated. Whole blood was obtained from healthy volunteers and surplus human hamstring tendon tissue was obtained during anterior cruciate ligament reconstruction via the Oxford Musculoskeletal Biobank with informed consent and in full compliance with UK HTA and MREC requirements.

2.1. Cell Culture. Primary human tenocytes were derived from healthy hamstring tendon explants, collected fresh from surgery. The cells were grown in bicarbonate-buffered DMEM: F12 (Cambrex, Wokingham, UK) with 10\% heatinactivated FCS (Biosera, Ringmer, UK), $100 \mathrm{U} / \mathrm{mL}$ penicillin, and $100 \mathrm{mg} / \mathrm{mL}$ streptomycin. For hypoxia experiments, the cells were cultured in $10 \%$ or $1 \%$ FCS-containing medium with $0.1 \%$ oxygen for the times indicated. The cells cultured in 10\% FCS with atmospheric oxygen served as control. Cells within three passages were used for all experiments except initial phenotyping experiments which were run out to 8 passages. Human myeloma cell line RPMI 8226 cells were cultured in RPMI medium and used as positive control for $\mathrm{Bcl} 2$ family protein expression and VEGF gene expression. Recombinant human VEGF B 167 [14] was obtained from R\&D systems (Abingdon, UK).

2.2. Western Blotting. Total cell protein was extracted from human tenocytes within three passages. Protein concentration was measured using the BCA protein assay kit (Pierce Biotechnology, Rockford, IL). Equal protein samples were separated on SDS-PAGE gels by electrophoresis and transferred onto a polyvinylidene fluoride (PVDF) membrane (Millipore, Bedford MA). Prestained Precision Plus molecular weight markers (BioRad, Hercules, CA) were used to estimate molecular weight. Rabbit polyclonal primary antibodies: anti-Bim and anti-cleaved Caspase-3 antibodies were purchased from Calbiochem (San Diego, CA); anti-Bax, antiBad, Puma, Bmf, Noxa antibodies were from Cell Signalling Technologies (Beverley, MA); anti-Bak antibody was from Upstate (Dundee, UK); Bcl2, Bcl-xL (Santa Cruz, CA), Nix (Axxora, Lausen, Switzerland). Mouse monoclonal primary 
antibodies: Bnip3 (Axxora, Lausen, Switzerland), HIF-1 $\alpha$ (BD Biosciences, Erembodegem, Belgium), $\beta$-Tubulin (Sigma, Poole, UK), FOXO-3A (Epitomics, Burlingame, CA). Secondary antibodies were goat anti-rabbit horseradishperoxidase conjugated antibody (Abcam, Cambridge, UK) and goat anti-mouse horseradish-peroxidase conjugated antibody (Pierce Biotechnology, Rockford, IL). Immunocomplexes were detected using enhanced chemiluminescence detection system or Supersignal West Dura extended duration substrate (Pierce Biotechnology, Rockford, IL) and signals were captured using a UVP Chemidoc-it. VisionWorksLS software v6.7.4 was used to measure the density of bands of interest. $\beta$-Tubulin was stable under hypoxic conditions and served as internal control.

2.3. Standard RT-PCR and Real-Time Quantitative RT-PCR. Total mRNA was isolated from cells using RNeasy Mini kits (Qiagen, GmbH, Hilden, Germany), according to the manufacturer's protocol. RNase-Free DNase Set (Qiagen, $\mathrm{GmbH}$, Hilden, Germany) was used to remove genomic DNA contamination. $0.3 \mu \mathrm{g}$ total RNA in a $20 \mu \mathrm{L}$ reaction volume was reverse-transcribed using SuperScript II Reverse Transcriptase (Invitrogen, Paisley, UK) in the presence of oligo-dT primer (Invitrogen, Paisley, UK). Samples without reverse transcriptase served as negative controls in real-time PCR reactions to exclude genomic DNA contamination. Notemplate control was always included to indicate that there was no contamination in real-time PCR reagents. Scleraxis and $\beta$-actin primers were Quantitect primers from Qiagen and other primers $[27,28]$ were from MWG Biotech (London, UK). Human VEGF-A primers to detect all splice variants, forward 5'-CTTGCCTTGCTGCTCTACC-3' (Exon 1) and reverse $5^{\prime}$-CACACAGGATGGCTTGAAG-3' (Exon 3); VEGF-A primers to distinguish different splice variants, forward 5'-CTCACCAAGGCCAGCACATAGG-3' (Exon 4), reverse $5^{\prime}$-ATCTGGTTCCCGAAACCCTGAG-3' (Exon 8). Human VEGF-B forward primer was $5^{\prime}$-TGTGTATACTCGCGCTACCTG-3' and reverse primer was $5^{\prime}$-CATTCACACTGGCTGTGTTC- ${ }^{\prime}$. Human VEGF-C forward primer was $5^{\prime}$-GTCTGTGTCCAGTGTAGATG- $3^{\prime}$ and reverse primer was $5^{\prime}$-AGGTAGCTCGTGCTGGTGTT-3'. Human VEGFD forward primer was $5^{\prime}$-CAGTGAAGCGATCATCTCAGTC-3' and reverse primer was $5^{\prime}$-TACGAGGTGCTGGTGTTCATAC- $3^{\prime}$. Standard PCR reactions were performed using a Thermo Hybaid system. The amplicons were separated by electrophoresis to confirm the size of the products. Real-time quantitative PCR reactions were performed using a Corbett Rotor-Gene 3000 using QuantiTect SYBR Green PCR kit (Qiagen, GmbH, Hilden, Germany). Samples were run in duplicate and the average value was used for analysis. Comparative Quantitation analysis [29, 30] (Corbett RotorGene version 6.0.1) was used to compare the gene expression of VEGF-A in treated samples relative to control cells and normalised to the housekeeping gene $\beta$-actin.

2.4. ELISA. Cell culture supernatants were collected from hypoxia treated samples or control samples and kept at $-80^{\circ} \mathrm{C}$ until use. VEGF-A expression was measured by using Quantikine Human VEGF ELISA kit, according to the manufacturer's protocol (R\&D Systems, Abingdon, UK). Absorbance was measured by microplate reader at $450 \mathrm{~nm}$. Results were normalized to total protein of the cells and data were presented as $\mu \mathrm{g}$ VEGF-A/ $\mu \mathrm{g}$ total protein.

2.5. Apoptosis Determination. An in situ cell death detection kit (Roche Applied Science, Penzberg, Germany) was used to perform the TUNEL reaction to identify apoptotic cells with fragmented DNA, according to the manufacturer's instructions. DAPI was used to visualise all nuclei. The cells were observed and photographed using an Olympus BX40 microscope and Olympus DP70 camera. TUNEL-and DAPIpositive cells were counted manually using cell ${ }^{\wedge} \mathrm{F}$ program (Olympus, Soft Imaging System GmbH, Münster, Germany) and 15-20 fields were taken from each slide.

2.6. Platelet Rich Plasma Treatment. Platelet Rich Plasma was generated from fresh whole human blood using Smith \& Nephew's Caption device. $55 \mathrm{~mL}$ from a healthy volunteer was collected directly into a syringe containing $5 \mathrm{~mL}$ acid citrate dextran anticoagulant. Platelets were concentrated according to Smith \& Nephew's protocol and stored frozen until use (freezing was found to reduce platelet activity and should be avoided where possible). $1 \mathrm{~mL}$ of PRP was activated with bovine thrombin and the resulting clots left to condition $9 \mathrm{~mL}$ of standard culture medium containing $1 \%$ FCS for $16 \mathrm{~h}$. Conditioned medium was centrifuged at $1400 \mathrm{~g}$ and syringe-filtered to remove debris prior to use. Hamstring tenocytes were cultured in 10\% FCS until 70\% confluence when medium was replaced with $1 \%$ FCS. Paired cultures were treated with or without $10 \%$ PRP conditioned medium and placed either in a multigas incubator set at $0.1 \%$ oxygen or under standard culture conditions of atmospheric oxygen for $48 \mathrm{~h}$. Cells were stained using Live \& Dead stain (Invitrogen, Paisley, UK) and imaged with an Olympus BX40 microscope and Olympus DP70 camera, followed by analysis with ImageJ software.

2.7. Statistical Analysis. Data are presented as means $\pm \mathrm{SD}$ or SEM from three or more separate experiments, as indicated. Data were analyzed using GraphPad Prism v 4.03 (GraphPad Software, Inc., La Jolla, CA). Student's $t$-test (for single comparison) or one-way ANOVA (for multigroup comparisons) with either Dunnett or Tukey post hoc test were used as appropriate. $P<0.05$ was regarded as denoting statistical significance.

\section{Results}

3.1. Characterisation of Normal Human Hamstring Tenocytes In Vitro. We have established a protocol for deriving large numbers of healthy human tenocytes from hamstring tendon. Hamstring tendon is used during routine anterior cruciate ligament reconstruction and the discarded stubs are collected from theatre, finely diced and explanted in tissue culture. Over a period of 2 weeks tenocytes migrate out of the explanted tissue pieces and can be amplified in culture. To assess phenotypic drift in our cultures mRNA was collected from tenocytes derived from 3 donors at passage 


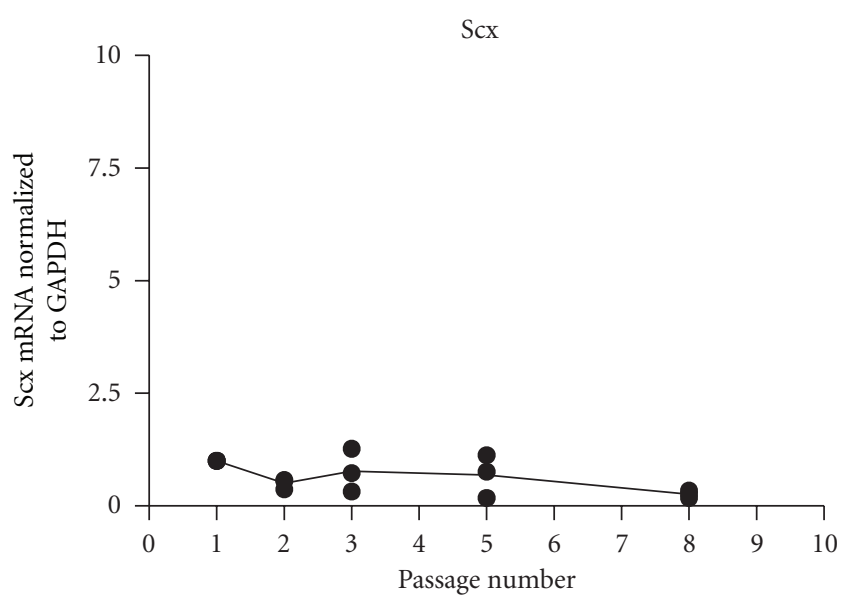

(a)

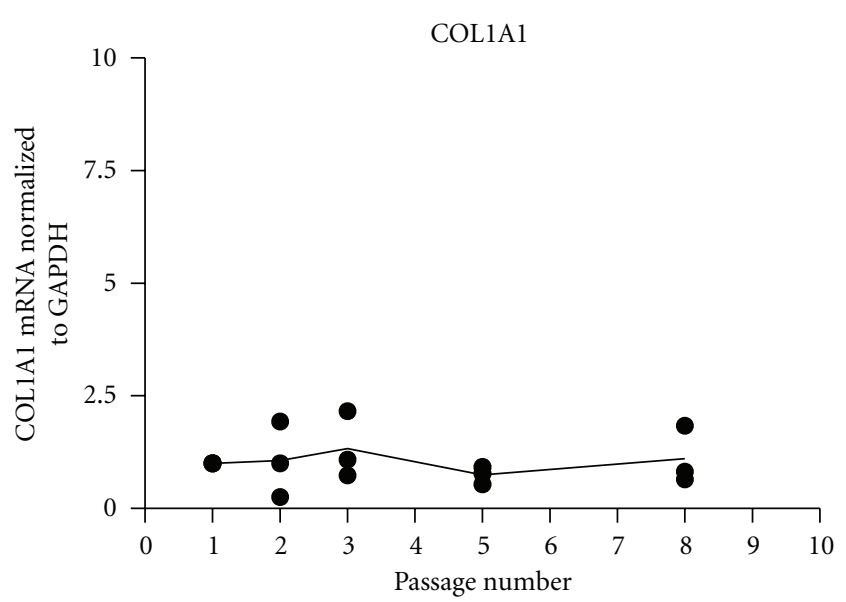

(b)

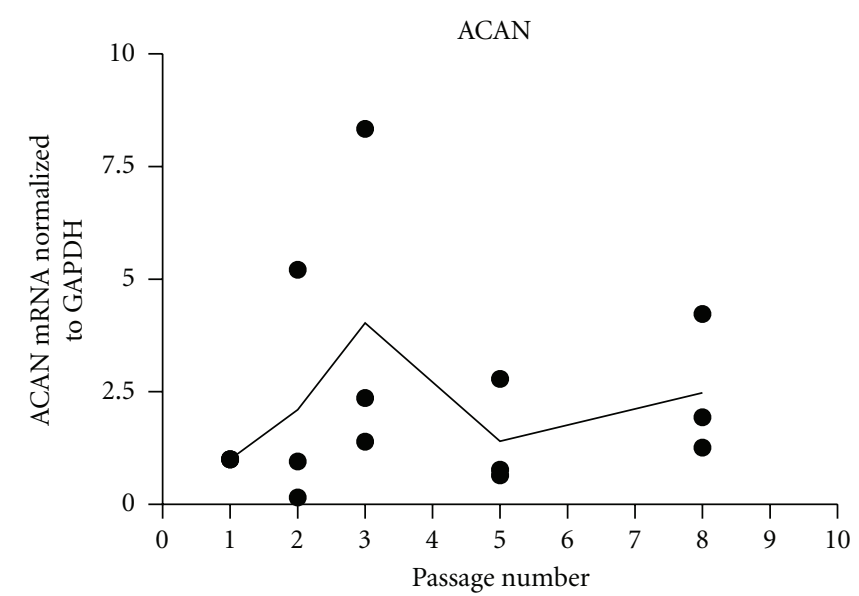

(c)

FIGURE 1: Human tenocytes express stable levels of scleraxis, collagen and aggrecan until passage 5 but scleraxis drops between passage 5 and 8 . Human tenocytes from 3 individual donors were cultured in 10\% FCS-containing medium under normoxia. (a) the expression of tenocyte ACAN, COL1A1, and Scx was quantified by rtq-PCR with $\beta$-actin as house-keeper.

$1,2,3,5$, and 8 and expression of collagen I, aggrecan core protein and the tenocyte transcription factor, scleraxis, were quantified (Figure 1). Although collagen I and aggrecan expression was relatively consistent, scleraxis levels dropped between passage 5 and 8 and therefore we have performed all tenocyte experiments on cells up to passage 3 .

\subsection{Hypoxia Rapidly Induces HIF-1 $\alpha$ Expression in Human} Tenocytes. To assess the response of human tenocytes to reduced oxygen, primary tenocytes were cultured in medium containing 10\% FCS under normoxic conditions until the cells reached $70 \%$ confluence. Medium was then changed to either $10 \%$ or $1 \%$ FCS and the cells were exposed to total hypoxia $\left(0.1 \% \mathrm{O}_{2}\right)$ for $1,4,8,16,24$, and $48 \mathrm{~h}$. The appropriate level of serum for primary tenocyte culture is unknown and since growth factors can protect against ischemia we compared the effect of hypoxia against a background of either high (10\%) or low (1\%) serum throughout this study. The cell lysates were prepared in HIF buffer [31]. Hypoxia inducible factor- $1 \alpha$ subunit (HIF-1 $\alpha$ ) was detected by Western blotting. HIF-1 $\alpha$ expression was below detectable levels under normal oxygen conditions in human tenocytes (Figure 2(a)) but accumulated rapidly with hypoxia $(0.1 \%$ $\mathrm{O}_{2}$ ) in either $10 \%$ or $1 \%$ FCS containing medium (Figures 2 (a) and 2(b)). HIF- $1 \alpha$ reached a maximal level between 1 and $8 \mathrm{~h}$. $\beta$-Tubulin protein is stable under hypoxic conditions and was used as internal control.

3.3. Human Tenocytes Express VEGF-A, B, and C but Not D $m R N A$. VEGF is classically upregulated in oxygen-starved tissue to restore the normal vasculature. To establish the VEGF profile of human tenocytes, total RNA was extracted from the tenocytes at passage 1 and gene expression of VEGF-A, B, C, D, and $\beta$-actin were determined by standard RT-PCR (Figure 3(a)). Human myeloma cell line RPMI was used as a positive control. Human tenocytes expressed VEGF-A (lane 2), B (lane 6), and C (lane 8) but not D (lane 10) mRNA. RPMI cells expressed VEGF-A (lane 3), $\mathrm{B}$ (lane 7), and D (lane 11) but not C (lane 9) mRNA. $\beta$ actin was used as internal control for tenocytes (lane 12) and 


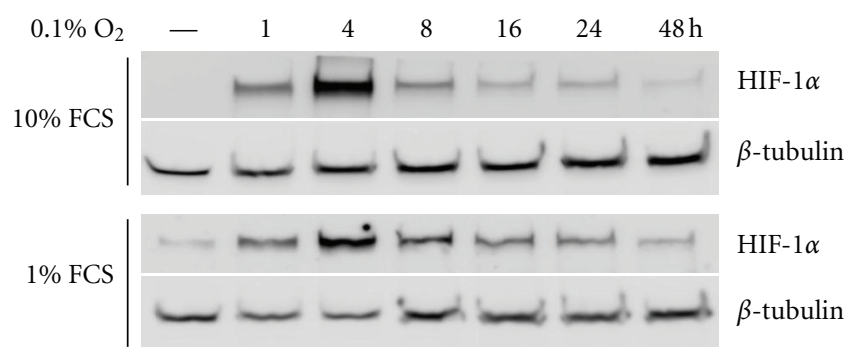

(a)

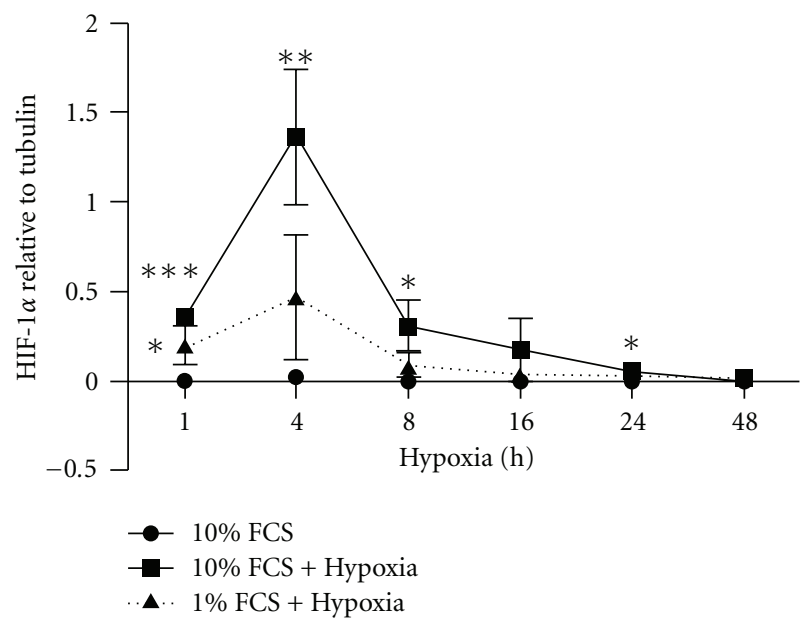

(b)

Figure 2: Hypoxia rapidly induces HIF- $1 \alpha$ expression in human tenocytes. Primary human tenocytes were cultured in DMEM/F12 culture medium containing 10\% FCS under normoxic conditions until the cells reached $70 \%$ confluence. The cells were exposed to total hypoxia $\left(0.1 \% \mathrm{O}_{2}\right)$ for $1,4,8,16,24$, and $48 \mathrm{~h}$. (a) Representative Western blot for HIF- $1 \alpha$ in tenocytes. (b) HIF- $1 \alpha$ expression qualified by densitometry is presented as mean \pm SE. $(n=3)$. ${ }^{*} P<0.05$ versus normoxic control; ${ }^{* *} P<0.01$ versus normoxic control; ${ }^{* *} P<0.001$ versus normoxic control.

RPMI (lane 13). Different VEGF-A splice variants were also detected using a primer pair that can separate the variants according to their different lengths. Both tenocytes (lane 4) and RPMI cells (lane 5) expressed four VEGF-A variants, including VEGF-121 (159 bp), 165 (291 bp), 189 (363 bp), and 206 (414 bp).

3.4. Both VEGF-A Gene and Protein Expression Are Upregulated by Hypoxia in Tenocytes. Following hypoxic exposure, relative VEGF-A gene expression was measured by real-time RT-PCR Figure 3(b)). VEGF-A gene expression was induced by hypoxia and reached a plateau before $24 \mathrm{~h}$. VEGF-A protein release was measured by ELISA by collecting the cell culture supernatants (Figure 3(c)). The VEGF-A antibody used recognizes all VEGF-A isoforms and the results were normalized to total protein of the cells. VEGF-A release in the culture medium increased $24 \mathrm{~h}$ after hypoxia treatment and further accumulated in the culture medium $48 \mathrm{~h}$ after hypoxia when the cells were cultured in either $10 \%$ or $1 \%$ FCS containing medium.
3.5. Bnip3 Is Upregulated by Hypoxia in Human Tenocytes. Another HIF-1alpha target gene is the proapoptotic Bcl2 family member, Bnip3 [11, 13]. Primary human tenocytes were exposed to total hypoxia $\left(0.1 \% \mathrm{O}_{2}\right)$ for $1,4,8,16$, 24 , and $48 \mathrm{~h}$. The cells were harvested in standard lysis buffer. Bnip3 is a $21.5 \mathrm{kDa}$ protein that typically migrates at either $35 \mathrm{kDa}$ (monomer) or $60 \mathrm{kD}$ (dimer) in SDS PAGE gels [32]. Multiple immuno-positive bands were recognized by Bnip3 antibody (Figure 4(a)), correlating with different Bnip3 isoforms [32]. Bnip3 expression was significantly increased at $8 \mathrm{~h}$ and $16 \mathrm{~h}$ after hypoxia treatment, slightly later than HIF- $1 \alpha$ induction (Figure 4(b)) and reached a peak between 8 and $24 \mathrm{~h}$ in human tenocytes (Figures 4(a) and $4(\mathrm{~b}))$.

Nix is a HIF-responsive Bnip3 homologue and was also found to be expressed in primary human tenocytes as a doublet at $37 \mathrm{kD}$ and $40 \mathrm{kD}[13,33]$. Nix $(37 \mathrm{kD}$ band) tended to increase at $16 \mathrm{~h}$ and $24 \mathrm{~h}$ after hypoxia, although there was no statistical significance (Figure 4(c)). Other proapoptotic Bcl2 family members Bim, Bak, Bax, and Puma were expressed in human tenocytes but remained unchanged throughout $48 \mathrm{~h}$ of hypoxia (data not shown). Noxa and Bmf expression was below detectable levels in human tenocytes but both were detected in the human myeloma cell line RPMI. Bad was also expressed in tenocytes. However, Bad expression was upregulated only by a combination of hypoxia with low serum in two out of three experiments, suggesting complex regulation (data not shown).

3.6. Antiapoptotic Proteins Bcl2 and Bcl-xL Remain at a Low Level after Hypoxia. The expression and regulation of antiapoptotic proteins $\mathrm{Bcl} 2$ and $\mathrm{Bcl}-\mathrm{xL}$ were determined by Western blotting after hypoxia. Both Bcl2 (Figure 4(d)) and $\mathrm{Bcl}-\mathrm{xL}$ (not shown) were expressed at low levels in tenocytes and remained unchanged after $48 \mathrm{~h}$ hypoxia.

3.7. Insulin Protects Tenocytes from Apoptosis Induced by Hypoxia. TUNEL and DAPI double-staining of cells grown on coverslips was used to evaluate apoptosis induced by hypoxia. Under normoxic conditions with 10\% FCS culture medium, tenocytes appeared healthy and spindle shaped (Figure 5(a), top left panel). Few TUNEL positive cells were observed, and DAPI staining showed normal morphology of nuclei. Serum starvation (1\% FCS) slightly increased TUNEL positive cells. However, hypoxia $\left(0.1 \% \mathrm{O}_{2}\right)$ significantly induced cell death from $1.6 \%$ under normoxia to $7.5 \%$ under hypoxia $48 \mathrm{~h}$ after treatment in human tenocytes (Figure 5(a), top middle panel and Figure 5(b)). Hypoxia combined with serum reduction (1\% FCS) further increased apoptosis by $19.5 \%$ after $48 \mathrm{~h}$ treatment (Figure 5(a), top right panel and Figure 5(b)). Since Akt/PKB activating growth factors are strongly antiapoptotic in many cell types we used high-dose insulin to block hypoxia-induced apoptosis. At $10 \mu \mathrm{g} / \mathrm{mL}$ or $30 \mu \mathrm{g} / \mathrm{mL}$ insulin treatment partially protected tenocytes from apoptosis in the presence of $10 \%$ FCS (Figure 5(a), bottom left panel and Figure 5(b)) or $1 \%$ FCS (Figure 5(a), bottom right panel and Figure 5(b)). In contrast, neither recombinant VEGF-B $(10-100 \mathrm{ng} / \mathrm{mL})$ 


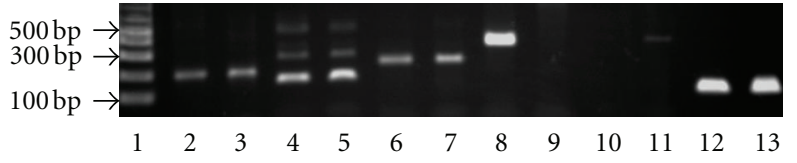

(a)

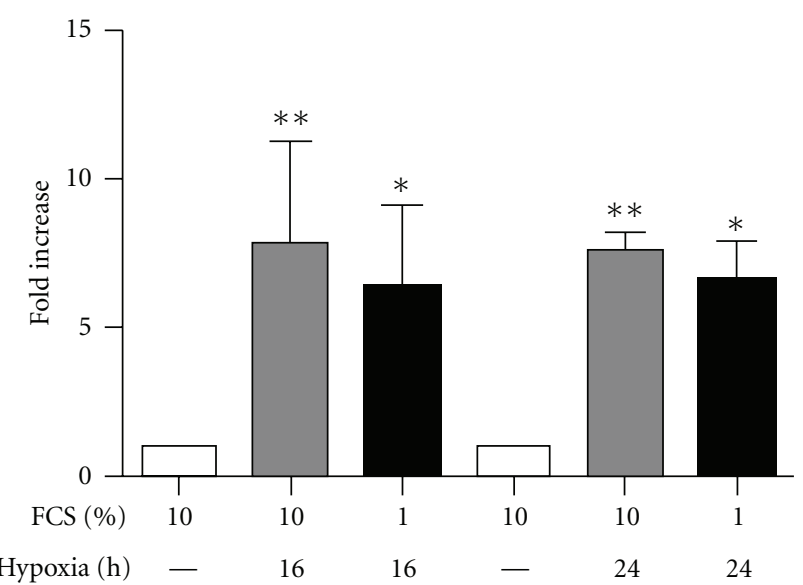

(b)

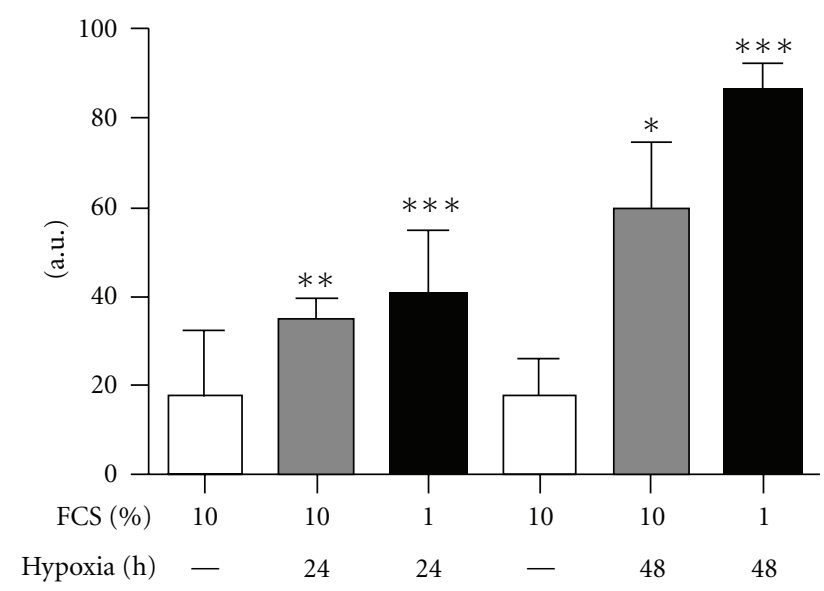

(c)

FIgure 3: Human tenocytes express VEGF-A, B, and C but not D mRNA and hypoxia treatment upregulates VEGF-A mRNA and protein expression. (a) Gene expression of VEGF-A (lane 2 and 3), B (lane 6 and 7), C (lane 8 and 9), D (lane 10 and 11), and VEGF-A splice variants (lane 4 and 5) was determined by standard RT-PCR with 35 cycles from human tenocytes (lane 2, 4, 6, 8, 10, and 12) at passage 1 or human myeloma cell RPMI (lane 3, 5, 7, 9, 11, and 13). Products were separated by electrophoresis. $\beta$-actin (lane 12 and 13) was used as housekeeping gene. (b) Hypoxia upregulates VEGF-A gene expression. Real-time quantitative PCR was used to compare the relative gene expression of VEGF-A 16 and $24 \mathrm{~h}$ after hypoxia and data are represented as fold change (mean $\pm \mathrm{SD}, n=3$; ${ }^{*} P<0.05$ or $* * P<0.01$ versus relevant time controls). (c) Hypoxia upregulates VEGF protein secretion. Cell culture supernatants were collected after hypoxia treatment for $24 \mathrm{~h}$ or $48 \mathrm{~h}$ and ELISA was used to measure VEGF-A secretion from human tenocytes. Data are represented as $\mu \mathrm{g}$ VEGF-A per $\mu \mathrm{g}$ total protein (mean $\pm \mathrm{SD}, n=3{ }^{*} P<0.05,{ }^{* *} P<0.01,{ }^{* * *} P<0.001$ versus relevant time control).

nor the stable, cell permeable cAMP analogue (8-(4Chlorophenyl)thio-cyclic AMP; $10-100 \mu \mathrm{M})$ demonstrated any protection (results not shown).

3.8. Insulin Does Not Decrease HIF1a Expression or Alter FOXO3A Expression. In order to examine the antiapoptotic mechanism by which insulin protects hypoxic tenocytes we assessed the levels of HIF1 $\alpha$ under normoxia and hypoxia. HIF $1 \alpha$ protein levels were not decreased by insulin treatment at $6 \mathrm{~h}$ (Figure 6(a)). The forkhead transcription factor FOXO3A has been reported to be upregulated in response to hypoxia [10], to strongly regulate Bnip3 [11] and to mediate damage responses such as autophagy $[11,34]$. We found that total hypoxia upregulated FOXO3A levels by $16 \mathrm{~h}$ in $10 \%$ but not $1 \%$ FCS (Figure 6(c)) and levels were still elevated after $48 \mathrm{~h}$ (Figure 6(d)). However, although insulin and other PKB activators have been described to oppose the actions of forkhead proteins we detected no decrease in FOXO3A protein levels with insulin and hypoxia cotreatment (Figures 6(b), 6(c), and 6(d)).

\subsection{Platelet Rich Plasma Protects Tenocytes from Hypoxia-} Induced Cell Death. Since insulin is not feasible for use as an in vivo therapy for tendon we tested platelet-rich plasma (PRP) as a source of growth factors. Culture medium conditioned overnight with 10\% PRP was applied to tenocytes prior to hypoxic exposure for $48 \mathrm{~h}$ as above. Live \& Dead staining showed no detectable cell death in normoxic cultures (Figures $7(\mathrm{a}), 7(\mathrm{~b})$, and $7(\mathrm{c})$ ) with or without insulin (Figure 7(b)) or PRP (Figure 7(c)). Following $48 \mathrm{~h}$ of 


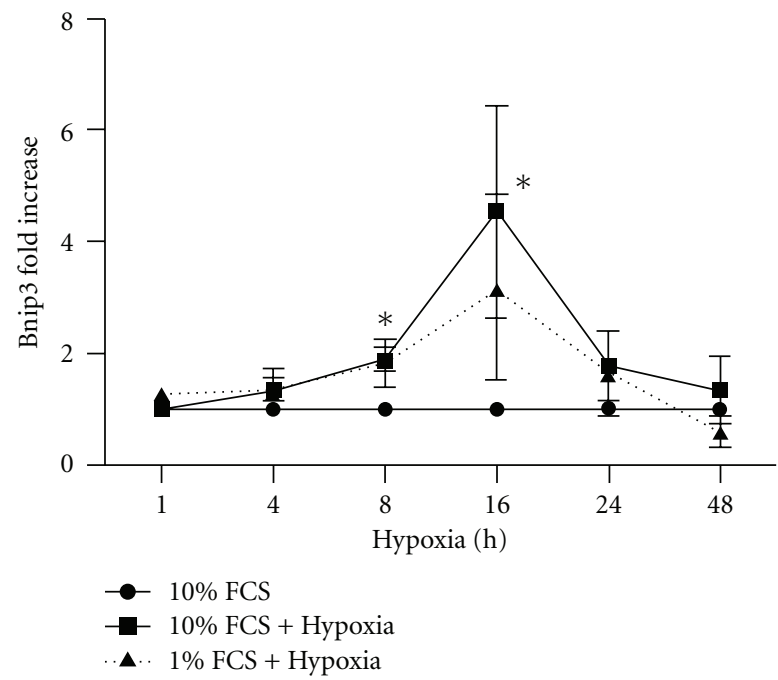

(a)

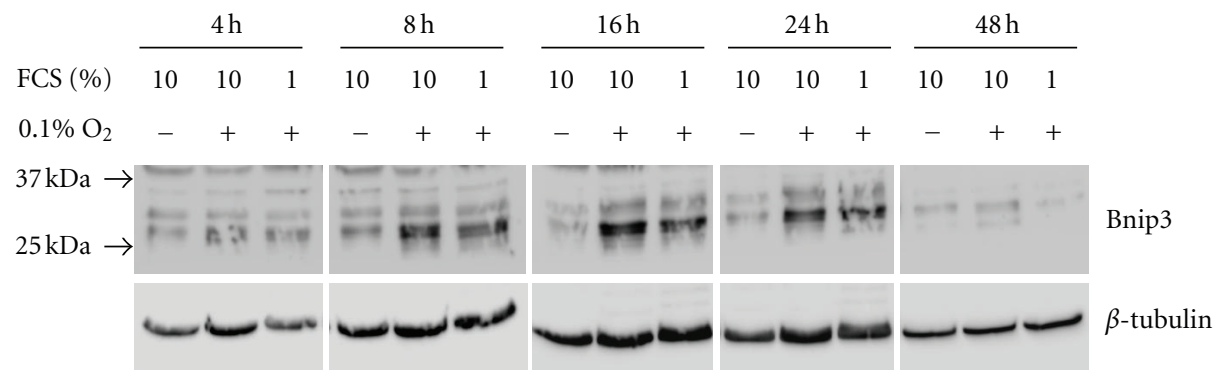

(b)

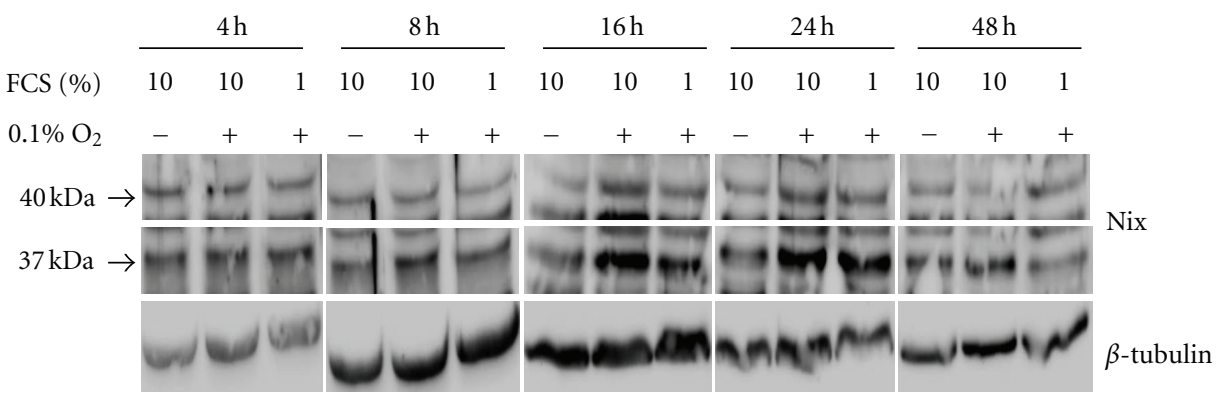

(c)

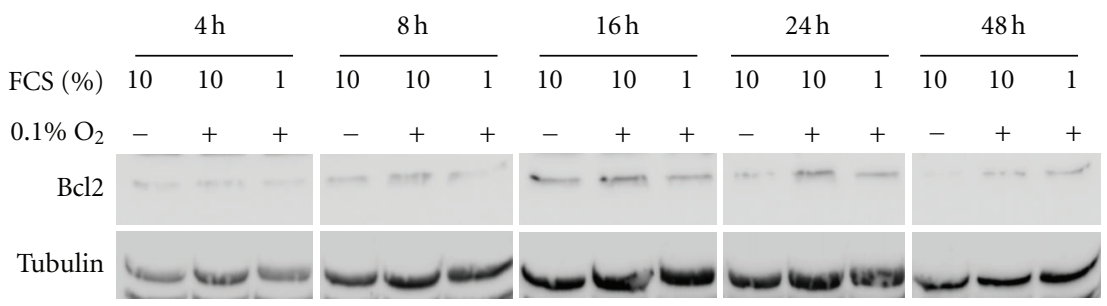

(d)

Figure 4: Bnip3 and Nix are upregulated by hypoxia in human tenocytes but Bcl2 is not. Primary human tenocytes were exposed to total hypoxia $\left(0.1 \% \mathrm{O}_{2}\right)$ for $1,4,8,16,24$, and $48 \mathrm{~h}$. The cells were harvested in cell lysis buffer. (a) Representative Western blot for Bnip3 in tenocytes. (b) Fold change of Bnip3 is presented as mean \pm SE $(n=3)$. * Represents $P<0.05$ versus control. (c) Representative western blot for Nix after hypoxia treatment for 1, 4, 8, 16, 24, and $48 \mathrm{~h}$ in tenocytes. (d) Antiapoptotic protein Bcl2 remains at low level after hypoxia treatment. Representative Western blot for Bcl 2 after hypoxia treatment for 1, 4, 8, 16, 24, and $48 \mathrm{~h}$ in tenocytes. 

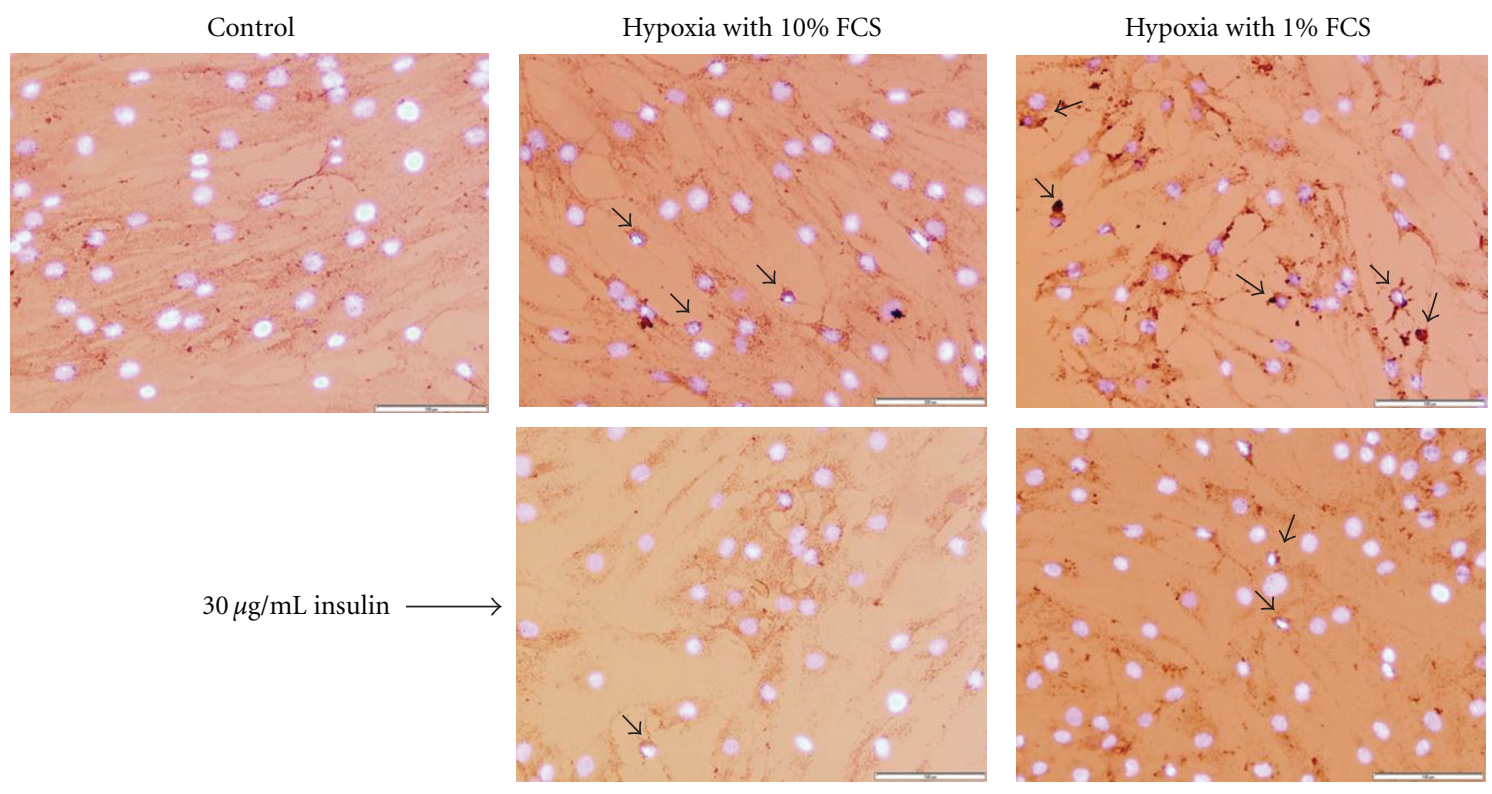

(a)

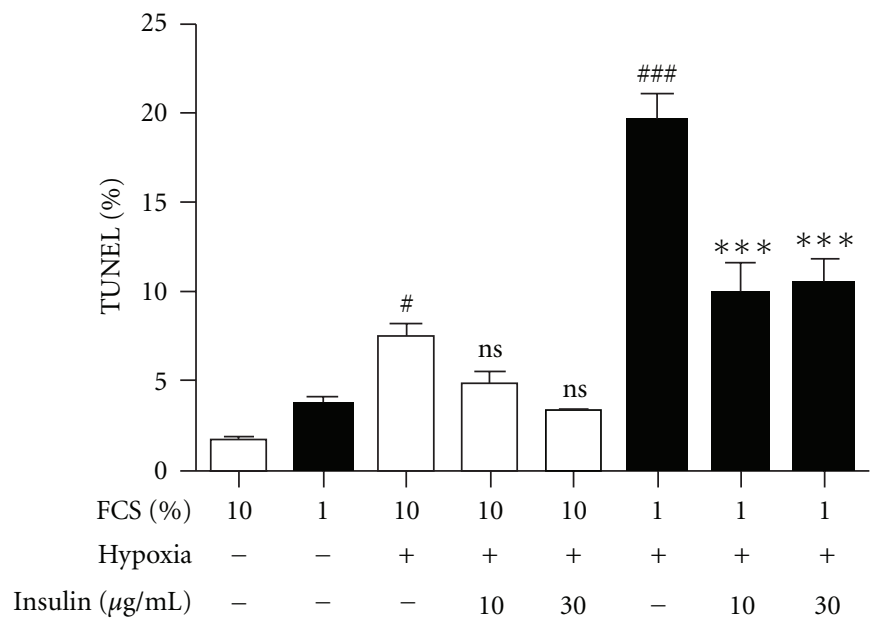

(b)

FIGURE 5: Insulin protects tenocytes from apoptosis induced by hypoxia. (a) TUNEL and DAPI double-staining from cells under normoxic control conditions (top left panel), hypoxia $\left(0.1 \% \mathrm{O}_{2}\right)$ treatment with $10 \%$ FCS for $48 \mathrm{~h}$ (top middle panel), hypoxia treatment with $1 \%$ FCS for $48 \mathrm{~h}$ (top right panel) and $30 \mu \mathrm{g} / \mathrm{mL}$ insulin treatment for $48 \mathrm{~h}$ with hypoxia in the presence of $10 \%$ FCS (bottom left panel) or $1 \%$ FCS (bottom right panel). (b) Percentage of apoptotic cells versus total cells (mean $\pm \mathrm{SE}, n=3$; ${ }^{\#} P<0.05$ versus $10 \%$ FCS alone; ${ }^{\# \#} P<0.001$ versus $1 \%$ FCS alone; ns not significant versus $10 \%$ FCS + hypoxia; $* * P<0.001$ versus $1 \%$ FCS + hypoxia).

hypoxia the ratio of dead to live cells was clearly increased (Figure $7(\mathrm{~d})$ ) and treatment with either insulin (Figure 7(e)) or PRP (Figure 7(f)) provided protection. PRP reduced the percentage of dead cells from $20 \%$ to $8 \%$ of total cells (Figure 7(g)).

\section{Discussion}

The biology of mature tenocytes differs from bone and skin fibroblasts, having more in common metabolically with cartilage. Tenocytes are capable of withstanding extreme mechanical conditions and reduced perfusion during sustained periods of loading. It is unclear whether tenocytes respond to low oxygen using the classical hypoxia response pathways or how vulnerable they are to acute hypoxic insult. We and others have noted a severe loss of cellularity in both ruptured and degenerating tendons and have recently described upregulation of HIF- $1 \alpha$ and the hypoxia-induced death gene Bnip3, correlating with increased apoptosis in ruptured rotator cuff tendon $[8,9]$. A clear understanding of the forces driving this loss of cells would allow development of effective protective strategies to enhance repair and recovery after trauma and also be of likely benefit during tendon graft integration.

We find that healthy human primary tenocytes respond to oxygen deprivation by rapidly upregulating protein levels 


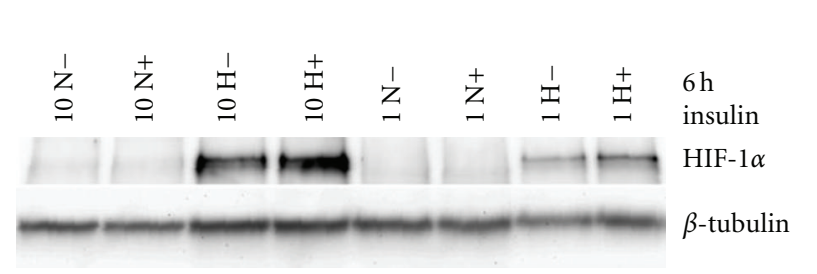

(a)

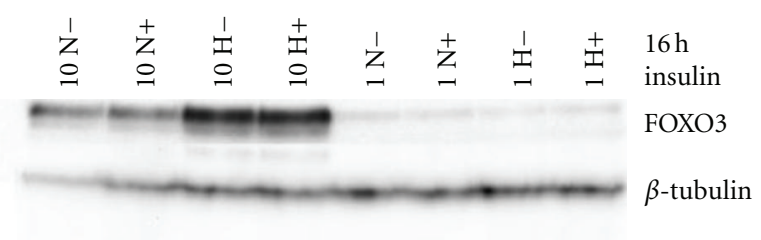

(c)

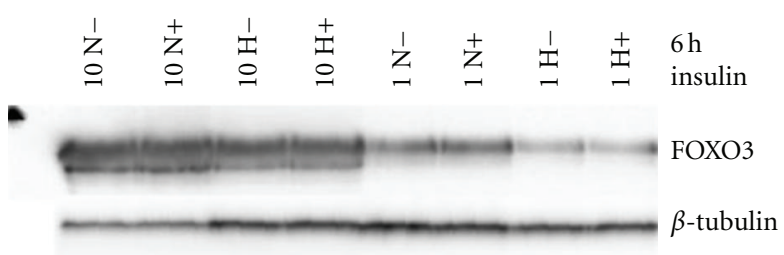

(b)

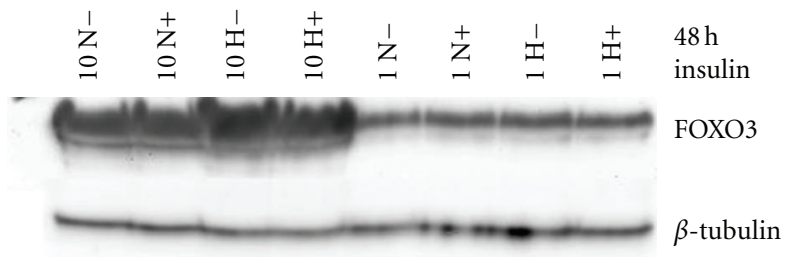

(d)

FIgURE 6: Cotreatment with insulin does not change the induction of HIF- $1 \alpha$ or FOXO-3A by hypoxia. Tenocytes subjected to $0.1 \%$ oxygen upregulated HIF-1 at $6 \mathrm{~h}$ in $10 \%$ FCS and less strongly in 1\% FCS. Cotreatment with $30 \mathrm{ug} / \mathrm{mL}$ insulin did not prevent the induction of HIF$1 \alpha$ (a). The stress responsive transcription factor FOXO3A was not changed after $6 \mathrm{~h}$ of hypoxia (b) but was upregulated at the later time points of $16 \mathrm{~h} \mathrm{(c)} \mathrm{and} 48 \mathrm{~h}(\mathrm{~d})$. Cotreatment with $30 \mathrm{ug} / \mathrm{mL}$ insulin had no effect on FOXO-3A protein levels at any time-point. Representative Western blots are shown from each time-point. Experiments were repeated with cells derived from 2 donors.

of the classical hypoxia responsive transcription factor HIF1 $\alpha$. This is followed within $48 \mathrm{~h}$ by significant apoptosis. Two hypoxia-associated proapoptotic proteins, Bnip3, and Nix were upregulated in response to hypoxia, with Bnip3 levels peaking 4 -fold higher then normoxic control between $8-16 \mathrm{~h}$. Both proteins have been described as HIF-1 $\alpha$ driven and their promoters contain hypoxia response elements [35]. Hypoxia did not downregulate the prosurvival proteins $\mathrm{Bcl} 2$ and Bcl-xL, nor was there any clear effect on other hypoxiarelevant proapoptotic proteins Bim, Bak, Bax, Puma, or Bad. Bmf and Noxa were not detectable in these tenocytes.

VEGF is an essential growth factor in the response of most tissues to traumatic injury involving disrupted blood supply. While VEGF induction has been reported in vivo following tendon rupture it has not been clear whether tenocytes themselves are mediating this in response to hypoxia, or other neighbouring cells including blood vessels. Also, induction by injury may be due to factors other than hypoxia since VEGF is also induced by proinflammatory cytokines and reactive oxygen species. We find that healthy human tenocytes express several isoforms of VEGF, including 4 splice variants of VEGF A as well as VEGF B and VEGF C. VEGF A is upregulated 8-fold at the mRNA level following hypoxia under both low (1\% FCS) and high (10\% FCS) serum conditions. Additionally, VEGF protein release into the culture medium is increased 4-fold by anoxia. This suggests that tenocytes display the typical mechanism by which hypoxic tissue attracts ingrowing blood vessels to restore normal perfusion following disruption due to injury.

Although VEGF induction is necessary for tissue repair, tendon pathology has also been associated with sustained elevation of VEGF $[3,36]$. This failure to resolve after healing is thought to be pathological and is currently being targeted using sclerosing drugs [3]. The outcome of VEGF elevation in rotator cuff stages is not known but our observation is that VEGF levels are lower than controls in the end-stage disease with large and massive tears [6]. Our data from healthy hamstring tenocytes suggests that VEGF elevation is a rapid and strong response to hypoxic insult, typical of most tissues. In the clinical setting chronic VEGF elevation once perfusion has been restored may be due to other factors such as inflammation and it will be important to study the contribution of acute versus chronic VEGF in tendon healing.

We found insulin and the growth factor cocktail released by PRP to be strongly antiapoptotic in hypoxic human hamstring tenocytes. Insulin dropped apoptosis levels to $50 \%$ and PRP to $40 \%$ of hypoxia induced levels in low serum conditions. In contrast VEGF or the commonly protective stable cAMP analogue cpt-cAMP were ineffective. This suggests that although VEGF is widely expressed in damaged tendons it is most likely of functional importance to other cell types such as endothelial cells. The protective effect of insulin was not due to prevention of upregulation of the hypoxiainduced transcription factors HIF $1 \alpha$ or FOXO3A. However, both of these transcription factors display complex regulation. HIF1 $\alpha$ stability and DNA-binding can be modified by multiple mechanisms [37]. In healthy or growth factorstimulated cells FOXO3A is phosphorylated by $\mathrm{PKB}$, causing its nuclear export and tethering to 14-3-3 adaptor protein in the cytoplasm [38]. Forkheads accumulate in the nucleus in response to JNK activation and when $\mathrm{PKB}$ activity drops [38]. The localization and DNA-binding activities of both HIF1 $\alpha$ and FOXO3A should therefore be studied following insulin and hypoxia treatment of healthy tenocytes to better understand the protective mechanism.

In summary, normal human tenocytes respond to complete oxygen deprivation by activating typical response pathways. HIF $1 \alpha$ and its target genes Bnip3 and VEGF are strongly upregulated and the damage response forkhead 


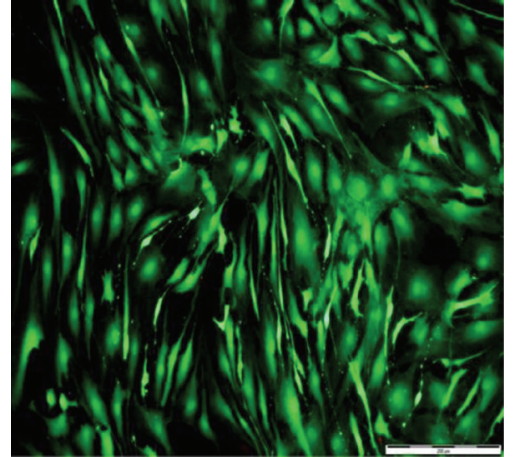

(a)

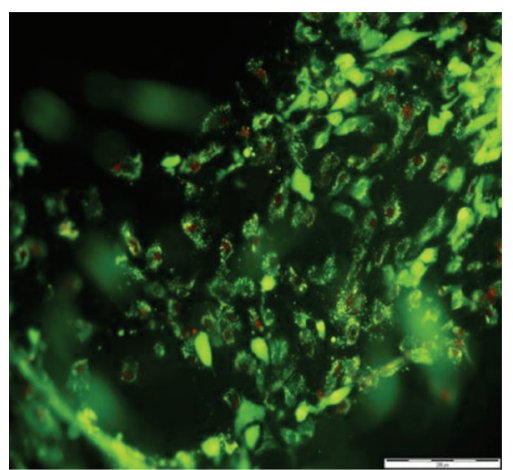

(d)

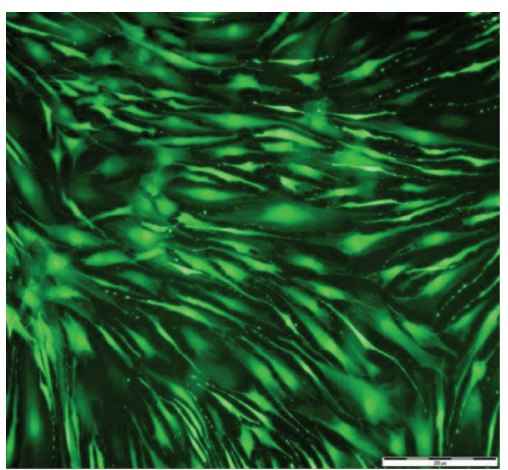

(b)

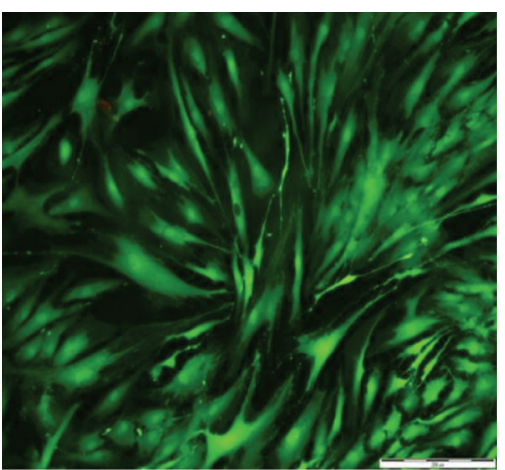

(e)

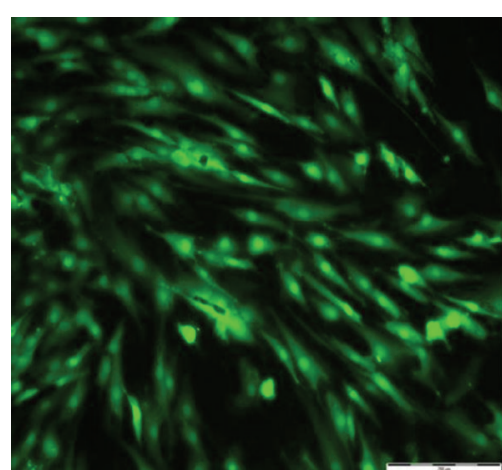

(c)

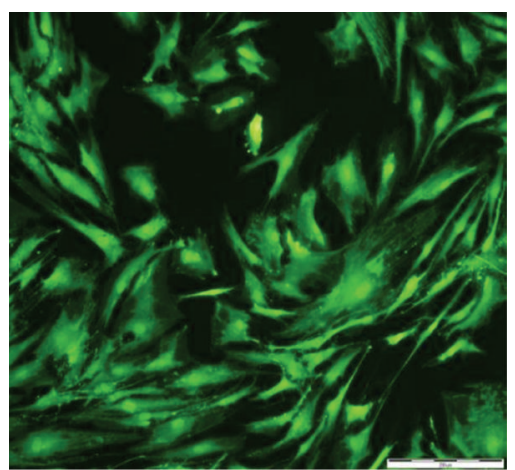

(f)

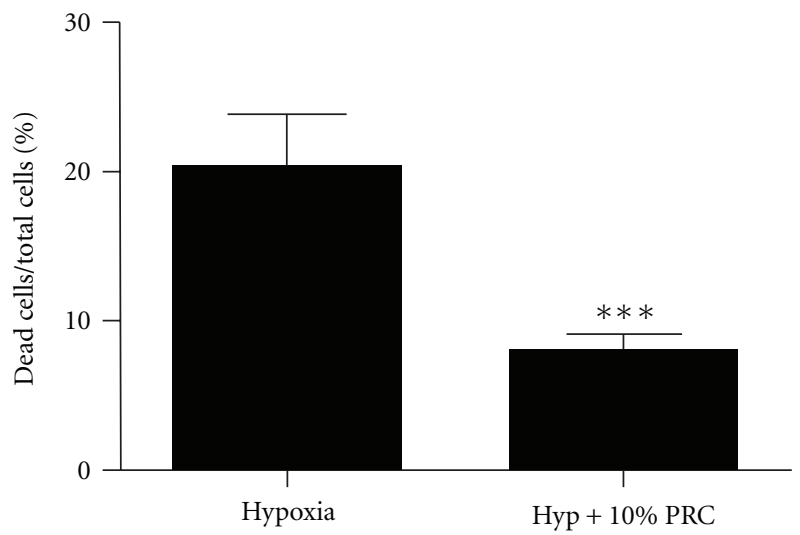

(g)

Figure 7: Platelet rich concentrate protects tenocytes against hypoxia-induced cell death. Live \& Dead staining of tenocytes following treatment of cells for $48 \mathrm{~h}$ under normoxia alone (a), with the addition of $30 \mathrm{ug} / \mathrm{mL}$ insulin (b) or $10 \%$ PRC conditioned medium (c) and following $48 \mathrm{~h}$ with $1 \%$ oxygen (d), with addition of $30 \mathrm{ug} / \mathrm{mL}$ insulin (e) or 10\% PRC conditioned medium (f). (g) Percentage of dead cells versus total cells with hypoxia treatment alone or in combination with $10 \% \operatorname{PRC}\left(\right.$ mean $\pm \mathrm{SD}, n=5$ ). ${ }^{* * *} P=0.0007$ versus hypoxia.

FOXO3A is also activated. Cell response was strongly modulated by the concentration of FCS, with reduced signaling under low serum conditions but considerably more apoptosis. This highlights the importance of perfusion and growth factors for the normal function of tendon, and the potential for increased susceptibility to cell death when tissue is disrupted by injury. Both insulin and PRP were effective in reducing hypoxia-induced cell death in cultured tenocytes, at least in the short term. PRP is highly practical as a rich source of autologous growth factors, since it can be extracted in theatre and reinjected into the same patient during repair or graft procedure. It is possible that application of these agents in the clinical setting may minimize hypoxia-induced damage to tendons in the clinical setting; however, future studies are indicated to more clearly define their role and efficacy clinically.

\section{Acknowledgments}

Smith and Nephew Ltd kindly provided the laboratory versions of their Caption device. Surgeons Mr Andrew Price and Mr Raj Rout are gratefully acknowledged for their 
assistance with tissue collection on behalf of the Oxford Musculoskeletal Biobank. Dr Helen Knowles is thanked for advice on HIF and VEGF detection methods. The Oxford Musculoskeletal BRU is acknowledged for infrastructure support. Contract grant sponsors of the paper are as follows: ENDO Stiftung (s-2/09); Lord Nuffield Orthopaedic Centre Trust, Arthritis Research UK with contract grant no. 19482, and Orthopaedic Research UK (formerly Furlong Research Charitable Foundation) with Contract grant no. 401.

\section{References}

[1] J. S. Sher, J. W. Uribe, A. Posada, B. J. Murphy, and M. B. Zlatkin, "Abnormal findings on magnetic resonance images of asymptomatic shoulders," Journal of Bone and Joint Surgery A, vol. 77, no. 1, pp. 10-15, 1995.

[2] J. B. Rathbun and I. Macnab, "The microvascular pattern of the rotator cuff," Journal of Bone and Joint Surgery B, vol. 52, no. 3, pp. 540-553, 1970.

[3] T. Pufe, W. J. Petersen, R. Mentlein, and B. N. Tillmann, “The role of vasculature and angiogenesis for the pathogenesis of degenerative tendons disease," Scandinavian Journal of Medicine and Science in Sports, vol. 15, no. 4, pp. 211-222, 2005.

[4] H. Yepes, M. Tang, S. F. Morris, and W. D. Stanish, "Relationship between hypovascular zones and patterns of ruptures of the quadriceps tendon," Journal of Bone and Joint Surgery A, vol. 90, no. 10, pp. 2135-2141, 2008.

[5] T. J. W. Matthews, G. C. Hand, J. L. Rees, N. A. Athanasou, and A. J. Carr, "Pathology of the torn rotator cuff tendon," Journal of Bone and Joint Surgery B, vol. 88, no. 4, pp. 489-495, 2006.

[6] R. T. Benson, J. L. Rees, P. A. Hulley, and A. J. Carr, "Vascular remodelling in rotator cufffailure," submitted.

[7] P. Sharma and N. Maffulli, "Tendon injury and tendinopathy: healing and repair," Journal of Bone and Joint Surgery A, vol. 87, no. 1, pp. 187-202, 2005.

[8] R. T. Benson, S. M. McDonnell, H. J. Knowles, J. L. Rees, A. J. Carr, and P. A. Hulley, "Tendinopathy and tears of the rotator cuff are associated with hypoxia and apoptosis," Journal of Bone and Joint Surgery B, vol. 92, no. 3, pp. 448-453, 2010.

[9] N. L. Millar, J. H. Reilly, S. C. Kerr et al., "Hypoxia: a critical regulator of early human tendinopathy," Annals of the Rheumatic Diseases, vol. 71, no. 2, pp. 302-310, 2012.

[10] W. J. Bakker, I. S. Harris, and T. W. Mak, "FOXO3a is activated in response to hypoxic stress and inhibits hif1-induced apoptosis via regulation of CITED2," Molecular Cell, vol. 28, no. 6, pp. 941-953, 2007.

[11] G. Chinnadurai, S. Vijayalingam, and S. B. Gibson, "BNIP3 subfamily BH3-only proteins: mitochondrial stress sensors in normal and pathological functions," Oncogene, vol. 27, no. 1, pp. S114-S127, 2008.

[12] T. Pufe, B. Kurz, W. Petersen et al., "The influence of biomechanical parameters on the expression of VEGF and endostatin in the bone and joint system," Annals of Anatomy, vol. 187, no. 5-6, pp. 461-472, 2005.

[13] H. R. Mellor and A. L. Harris, "The role of the hypoxia-inducible BH3-only proteins BNIP3 and BNIP3L in cancer," Cancer and Metastasis Reviews, vol. 26, no. 3-4, pp. 553-566, 2007.

[14] Y. Li, F. Zhang, N. Nagai et al., "VEGF-B inhibits apoptosis via VEGFR-1-mediated suppression of the expression of BH3only protein genes in mice and rats," Journal of Clinical Investigation, vol. 118, no. 3, pp. 913-923, 2008.
[15] C. G. Zou, X. Z. Cao, Y. S. Zhao et al., "The molecular mechanism of endoplasmic reticulum stress-induced apoptosis in PC-12 neuronal cells: the protective effect of insulin-like growth factor I," Endocrinology, vol. 150, no. 1, pp. 277-285, 2009.

[16] A. Scott, K. M. Khan, and V. Duronio, "IGF-I activates PKB and prevents anoxic apoptosis in Achilles tendon cells," Journal of Orthopaedic Research, vol. 23, no. 5, pp. 1219-1225, 2005.

[17] P. Hulley, J. Hartikka, S. Abdel'Al et al., "Inhibitors of type IV phosphodiesterases reduce the toxicity of MPTP in substantia nigra neurons in vivo," European Journal of Neuroscience, vol. 7, no. 12, pp. 2431-2440, 1995.

[18] H. T. Lee, Y. C. Chang, Y. F. Tu, and C. C. Huang, "VEGFA/VEGFR-2 signaling leading to cAMP response elementbinding protein phosphorylation is a shared pathway underlying the protective effect of preconditioning on neurons and endothelial cells," Journal of Neuroscience, vol. 29, no. 14, pp. 4356-4368, 2009.

[19] A. Rukenstein, R. E. Rydel, and L. A. Greene, "Multiple agents rescue PC12 cells from serum-free cell death by translationand transcription-independent mechanisms," Journal of Neuroscience, vol. 11, no. 8, pp. 2552-2563, 1991.

[20] J. Alsousou, M. Thompson, P. Hulley, A. Noble, and K. Willett, "The biology of platelet-rich plasma and its application in trauma and orthopaedic surgery: a review of the literature," Journal of Bone and Joint Surgery B, vol. 91, no. 8, pp. 987-996, 2009.

[21] M. De Mos, A. E. van der Windt, H. Jahr et al., "Can plateletrich plasma enhance tendon repair? A cell culture study," American Journal of Sports Medicine, vol. 36, no. 6, pp. 1171$1178,2008$.

[22] E. Anitua, I. Andía, M. Sanchez et al., "Autologous preparations rich in growth factors promote proliferation and induce VEGF and HGF production by human tendon cells in culture," Journal of Orthopaedic Research, vol. 23, no. 2, pp. 281-286, 2005.

[23] E. Anitua, M. Sánchez, M. M. Zalduendo et al., "Fibroblastic response to treatment with different preparations rich in growth factors," Cell Proliferation, vol. 42, no. 2, pp. 162-170, 2009.

[24] L. V. Schnabel, H. O. Mohammed, B. J. Miller et al., "Platelet Rich Plasma (PRP) enhances anabolic gene expression patterns in flexor digitorum superficialis tendons," Journal of Orthopaedic Research, vol. 25, no. 2, pp. 230-240, 2007.

[25] P. Aspenberg, "Stimulation of tendon repair: mechanical loading, GDFs and platelets. a mini-review," International Orthopaedics, vol. 31, no. 6, pp. 783-789, 2007.

[26] Y. Kajikawa, T. Morihara, H. Sakamoto et al., "Plateletrich plasma enhances the initial mobilization of circulationderived cells for tendon healing," Journal of Cellular Physiology, vol. 215, no. 3, pp. 837-845, 2008.

[27] P. O-charoenrat, P. Rhys-Evans, H. Modjtahedi, and S. Eccles, "Vascular endothelial growth factor family members are differentially regulated by c-erbB signaling in head and neck squamous carcinoma cells," Clinical and Experimental Metastasis, vol. 18, no. 2, pp. 155-161, 2000.

[28] T. Niki, S. Iba, M. Tokunou, T. Yamada, Y. Matsuno, and S. Hirohashi, "Expression of vascular endothelial growth factors A, B, C, and D and their relationships to lymph node status in lung adenocarcinoma," Clinical Cancer Research, vol. 6, no. 6, pp. 2431-2439, 2000.

[29] K. Warton, N. C. Foster, W. A. Gold, and K. K. Stanley, "A novel gene family induced by acute inflammation in endothelial cells," Gene, vol. 342, no. 1, pp. 85-95, 2004. 
[30] M. Liang, G. Russell, and P. A. Hulley, "Bim, Bak, and Bax regulate osteoblast survival," Journal of Bone and Mineral Research, vol. 23, no. 5, pp. 610-620, 2008.

[31] H. J. Knowles, R. R. Raval, A. L. Harris, and P. J. Ratcliffe, "Effect of ascorbate on the activity of hypoxia-inducible factor in cancer cells," Cancer Research, vol. 63, no. 8, pp. 1764-1768, 2003.

[32] R. M. Graham, J. W. Thompson, J. Wei, N. H. Bishopric, and K. A. Webster, "Regulation of Bnip3 death pathways by calcium, phosphorylation, and hypoxia-reoxygenation," Antioxidants and Redox Signaling, vol. 9, no. 9, pp. 1309-1315, 2007.

[33] A. Diwan, S. J. Matkovich, Q. Yuan et al., "Endoplasmic reticulum-mitochondria crosstalk in NIX-mediated murine cell death," Journal of Clinical Investigation, vol. 119, no. 1, pp. 203-212, 2009.

[34] J. Zhao, J. J. Brault, A. Schild et al., "FoxO3 coordinately activates protein degradation by the autophagic/lysosomal and proteasomal pathways in atrophying muscle cells," Cell Metabolism, vol. 6, no. 6, pp. 472-483, 2007.

[35] D. J. Manalo, A. Rowan, T. Lavoie et al., “Transcriptional regulation of vascular endothelial cell responses to hypoxia by HIF1," Blood, vol. 105, no. 2, pp. 659-669, 2005.

[36] T. Molloy, Y. Wang, and G. A. C. Murrell, "The roles of growth factors in tendon and ligament healing," Sports Medicine, vol. 33, no. 5, pp. 381-394, 2003.

[37] A. L. Bacon and A. L. Harris, "Hypoxia-inducible factors and hypoxic cell death in tumour physiology," Annals of Medicine, vol. 36, no. 7, pp. 530-539, 2004.

[38] D. R. Calnan and A. Brunet, "The FoxO code," Oncogene, vol. 27, no. 16, pp. 2276-2288, 2008. 


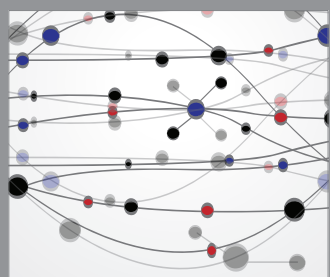

The Scientific World Journal
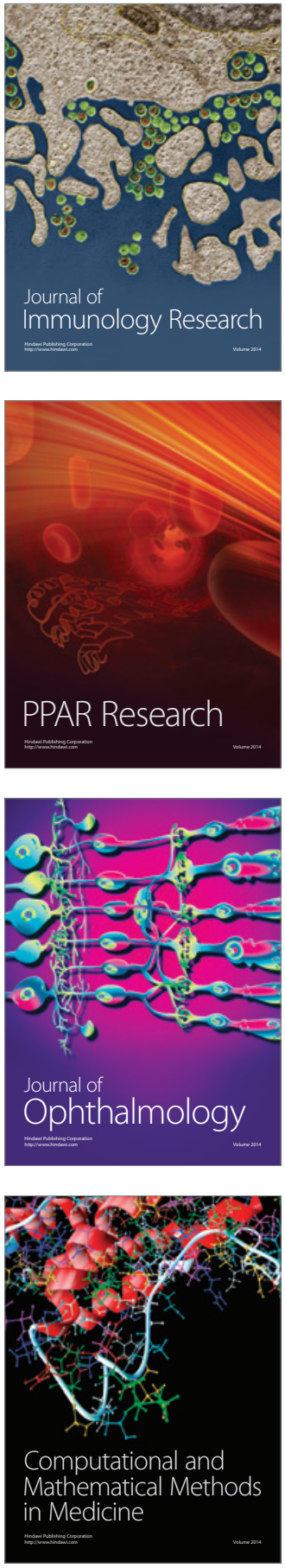

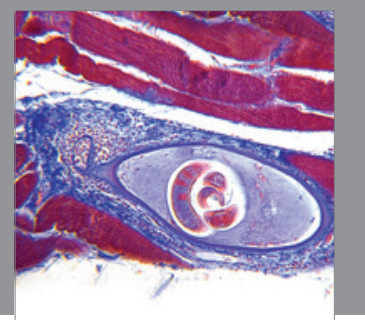

Gastroenterology

Research and Practice
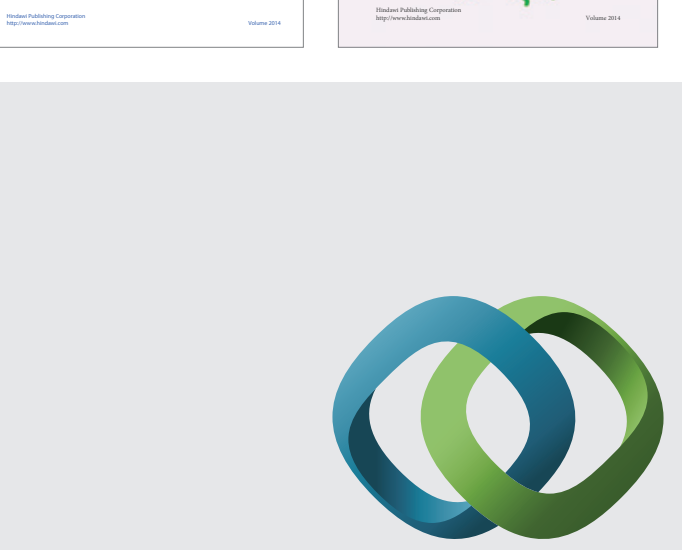

\section{Hindawi}

Submit your manuscripts at

http://www.hindawi.com
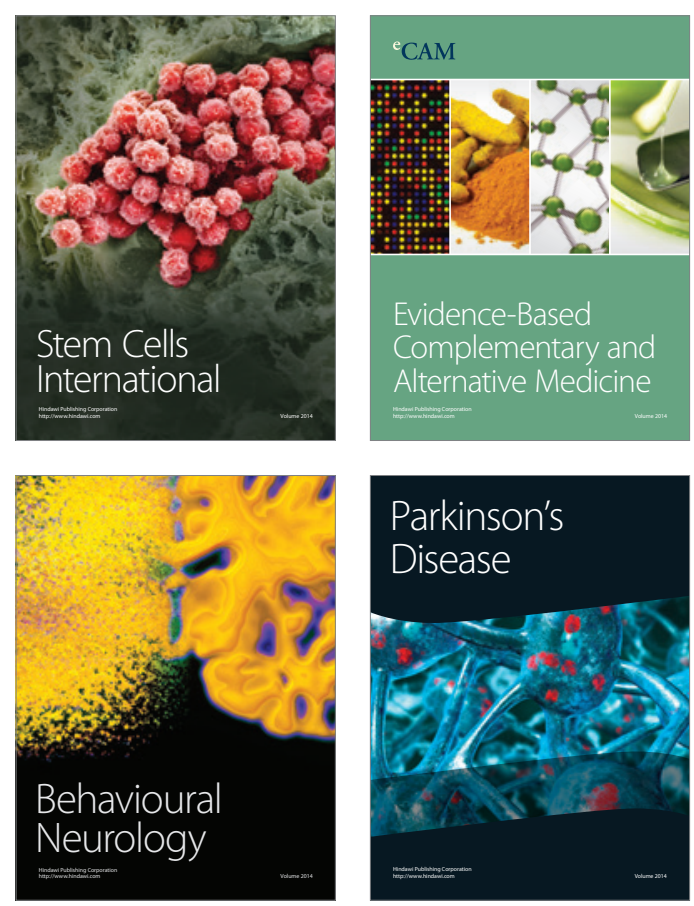

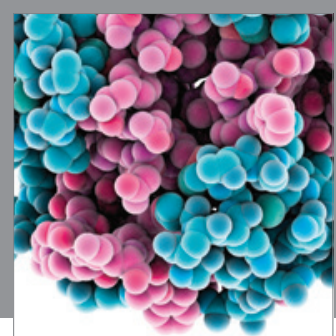

Journal of
Diabetes Research

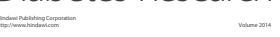

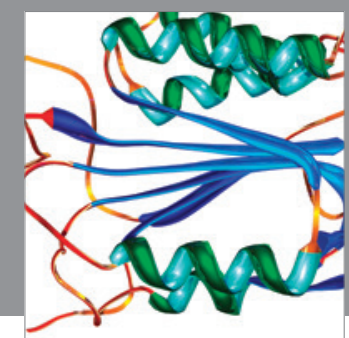

Disease Markers
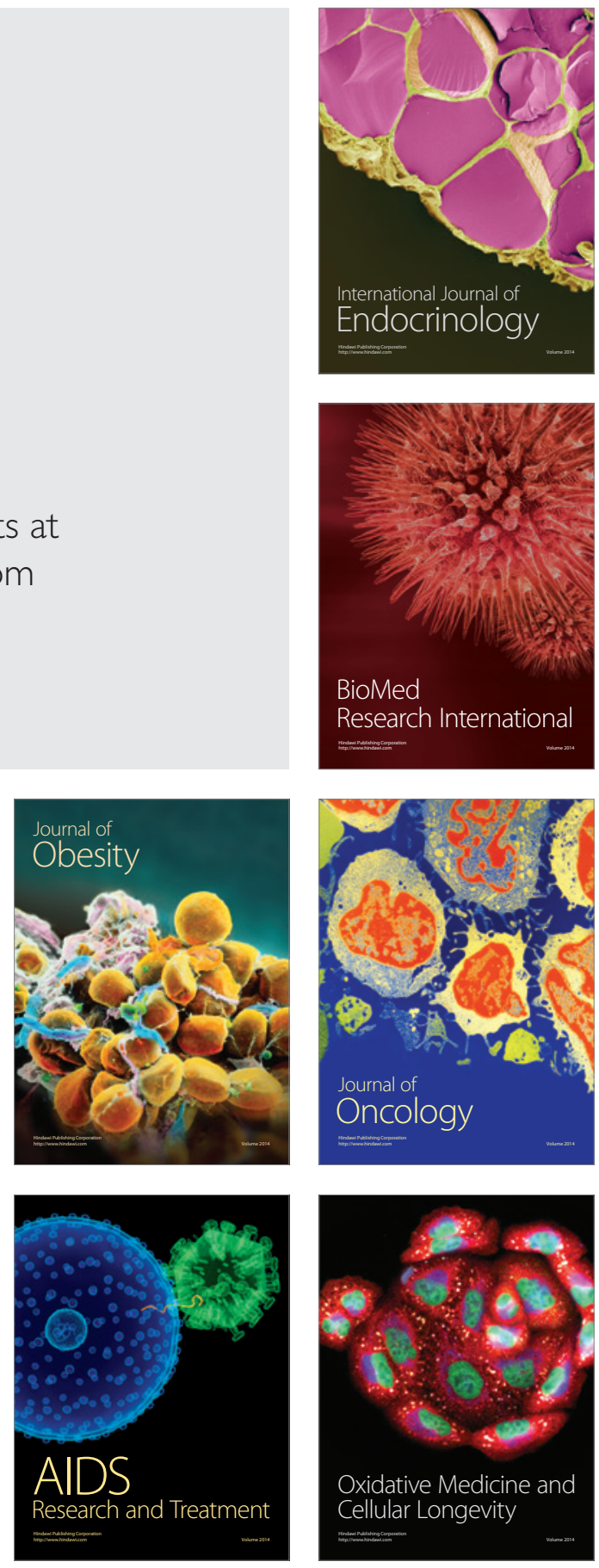